\title{
The effect of decompressive craniectomy on CSF pulsatility in experimental communicating hydrocephalus
}

\author{
Mark E Wagshul ${ }^{1 *}$, Shams Rashid ${ }^{2}$, James P McAllister $\|^{3}$, Martin U Schuhmann ${ }^{4}$ \\ From 54th Annual Meeting of the Society for Research into Hydrocephalus and Spina Bifida \\ Vancouver, Canada. 7-10 July 2010
}

\section{Background}

It has been well established that intracranial pulsatility, whether measured as parenchymal/intraventricular pressure or as cerebrospinal fluid (CSF) flow in the cerebral aqueduct, is often markedly elevated in patients with hydrocephalus. Attempts have been made to use this measure to predict outcome from shunt surgery, but its specificity is still questionable. The question of whether or not intracranial pulsatility plays a causative role in hydrocephalus or is merely an epiphenomenon related to intracranial compliance is similarly an unanswered question. As a first step toward answering this question, we hypothesized that aqueductal pulsatility would be reduced after increasing compliance following decompressive craniectomy.

\section{Materials and methods}

Communicating hydrocephalus was induced by injection of kaolin into the basal cisterns in five $(n=5)$ adult Sprague-Dawley rats; controls received similar saline injections. Flow pulsatility was studied at two weeks post-injection by cine phase contrast MRI prior to and immediately following bilateral craniectomy. Aqueductal stroke volume before and after craniectomy were compared. To minimize effects of extended anesthesia, the craniectomy was started prior to the first imaging scan without breaking through the bone completely. Animals were then moved to the scanner, imaged for the precraniectomy pulsatility measurements, and returned to the bench to complete the craniectomy. A bone flap
(4 x $10 \mathrm{~mm}$ ) was taken from each side over the parietal lobe. The dura mater was left intact, although small tears in the dura were noticed in some cases. A second scan was then obtained to measure post-craniectomy pulsatility. To ensure similar physiological state for the two scans, animals were intubated throughout and ventilated on $\mathrm{O} 2$ /isoflurane. Following the second scan, the animals were euthanized.

\section{Results}

All animals demonstrated moderate-to-severe ventriculomegaly (volumes from 111 - 322 microliters) and aqueductal pulsatility was elevated significantly in all cases compared to expected controls levels. In 4/5 animals, pulsatility decreased following craniectomy with a large variability in the percent reduction (17- 75 percent); the fifth animal exhibited no change. The animal with the largest decrease was also the most hydrocephalic and had the largest pre-craniectomy pulsatility.

\section{Conclusions}

These preliminary findings appear to be the first attempt to measure CSF pulsatility while manipulating intracranial compliance experimentally. The trend toward an immediate decrease in aqueductal pulsatility suggests that compliance plays an important role in CSF pulsatility. Further studies on the effects of shunting and/or CSF infusion should be performed in order to validate this mechanism.

\footnotetext{
* Correspondence: mark.wagshul@einstein.yu.edu

${ }^{1}$ Radiology, Gruss Magnetic Resonance Research Center, Albert Einstein

College of Medicine, Bronx, NY 10461, USA

Full list of author information is available at the end of the article
}

(c) 2010 Wagshul et al; licensee BioMed Central Ltd. This is an open access article distributed under the terms of the Creative Commons 


\section{Acknowledgement}

This work was supported by the BrainChild Foundation, Cave Creek, AZ, USA.

\section{Author details}

'Radiology, Gruss Magnetic Resonance Research Center, Albert Einstein College of Medicine, Bronx, NY 10461, USA. Biomedical Engineering, Stony Brook University, Stony Brook, NY 11794, USA. ${ }^{3}$ Pediatric Neurosurgery, University of Utah, Salt Lake City, UT 84132, USA. ${ }^{4}$ Pediatric Neurosurgery, University of Tubingen, Tubingen, Germany.

Published: 15 December 2010

doi:10.1186/1743-8454-7-S1-S35

Cite this article as: Wagshul et al: The effect of decompressive craniectomy on CSF pulsatility in experimental communicating hydrocephalus. Cerebrospinal Fluid Research 2010 7(Suppl 1):S35.

Submit your next manuscript to BioMed Central and take full advantage of:

- Convenient online submission

- Thorough peer review

- No space constraints or color figure charges

- Immediate publication on acceptance

- Inclusion in PubMed, CAS, Scopus and Google Scholar

- Research which is freely available for redistribution

Submit your manuscript at www.biomedcentral.com/submit 